\title{
EVALUACIÓN URODINÁMICA DE LA EFICACIA DE LA TOXINA BOTULÍNICA EN EL DETRUSOR HIPERACTIVO NEUROGÉNICO REFRACTARIO
}

\author{
Gustavo L. Garrido, Andrés Medrano, Edgardo Becher y Osvaldo Mazza.
}

División Urología del Hospital de Clínicas José de San Martín (HCSM). Universidad de Buenos Aires y CDU Centro de Urología. Buenos Aires. Argentina.

\begin{abstract}
Resumen.- OBJETIVOS: Del correcto tratamiento del detrusor hiperactivo neurogénico dependen aspectos fundamentales para la vida como la suficiencia renal hasta aspectos de importancia relevante para la calidad de vida como la incontinencia urinaria. Los fármacos anticolinérgicos permiten el tratamiento en la mayoría de los casos, pero su fracaso en el detrusor hiperactivo neurogénico refractario constituye un desafío. Evaluar de la eficacia de la inyección intradetrusorial de toxina botulínica tipo A en pacientes con detrusor hiperactivo neurogénico refractario, en los siguientes parámetros urodinámicos: capacidad cistomanométrica máxima, acomodación del detrusor, volumen de reflejo, presión máxima del detrusor y residuo postmiccional.
\end{abstract}

Gustavo L. Garrido Libertad 1985 Florida. Pcia. de Buenos Aires. Código Postal B 1 602DJl. (Argentina). ggarrido@intramed.net.ar

Trabajo recibido: 1 de agosto 2007.
MÉTODOS: Estudio prospectivo en 16 pacientes con diagnóstico de detrusor hiperactivo neurogénico refractario a la terapia anticolinérgica. Se realizó investigación urodinámica 30 a 60 días previos al procedimiento y luego de la interrupción de por lo menos 2 semanas de la terapia anticolinérgica. Se realizó la inyección endoscópica de 300 U de Toxina Botulínica tipo A (BTX-A) en 30 sitios de punción, a 10 unidades por $\mathrm{ml}$ por punción. Se realizó el control urodinámico 30 a 45 días posteriores a la inyección.

RESULTADOS: De los 16 pacientes estudiados 5 eran mujeres y 11 hombres, la edad media fue de 39,5 años (22 a 63 años). Los resultados urodinámicos hallados fueron: Capacidad cistomanométrica máxima media pre BTX-A fue de $204.73 \mathrm{ml}$ y la posterior fue de $381.87 \mathrm{ml}$. La presión máxima del detrusor media previa fue de $91.46 \mathrm{~cm} \mathrm{H} 2 \mathrm{O}$ mientras que la posterior fue $41.2 \mathrm{~cm} \mathrm{H2O}$. La presión de fin de llenado media previa fue de $68 \mathrm{~cm} \mathrm{H} 2 \mathrm{O}$ y la posterior $34 \mathrm{~cm}$ H2O. El volumen reflejo medio previo fue de 111.33 $\mathrm{ml}$, mientras que el posterior fue de $310.8 \mathrm{ml}$. Por último el residuo postmiccional pre-BTX-A medio fue de 129.6 $\mathrm{ml}$ mientras que el post-BTX-A de $345.9 \mathrm{ml}$.

CONCLUSIONES: La inyección de $300 \mathrm{U}$ de toxina botulínica tipo $\mathrm{A}$ en el detrusor ha demostrado ser eficaz y segura para el tratamiento del detrusor hiperactivo neurogénico refractario. En los parámetros urodinámicos se observaron diferencias estadísticamente significativas antes y después de la inyección. No se presentaron efectos adversos significativos.

Palabras clave: Toxina Botulínica. Vejiga neurogénica. Detrusor hiperactivo neurogénico. Detrusor hiperactivo neurogénico refractario. Inyección intradetrusorial. 
Summary.- OBJECTIVES: Proper treatment of neurogenic hyperactive detrusor muscle is responsible for several features essential for life such as renal function, and quality of life relevant issues such as urinary incontinence. Anticholinergic drugs allow treatment in most cases, but their failure in cases of refractory neurogenic hyperactive detrusor is a challenge. To evaluate the efficacy of detrusor injection of type $A$ botulin toxin in patients with refractory neurogenic hyperactive detrusor, on the following urodynamic parameters: maximum cystomanometric capacity, detrusor compliance, reflex volume, maximum detrusor pressure and post void residual.

METHODS: Prospective study in 16 patients with the diagnosis of neurogenic hyperactive detrusor refractory to anticholinergic therapy. Urodynamic studies were performed 30 and 60 days before the procedure and after at least two weeks from stopping anticholinergic therapy. 300 units of type A botulin toxin (BTX-A) were endoscopically injected in 30 punction sites, 10 units per $\mathrm{ml}$ each punction. Urodynamic control was performed 30 to 45 days after injection.

RESULTS: Six of the 16 patients in the study where females and 11 males; mean age was 39.5 years 122 to 63 years). Urodynamic results: mean maximum capacity on cystomanometry before BTX A was $204.73 \mathrm{ml}$ and after BTX-A $381.87 \mathrm{ml}$. Maximum detrusor capacity before was $91.46 \mathrm{H} 2 \mathrm{O} \mathrm{cm}$ whereas post-treatment was 41.2 $\mathrm{H} 2 \mathrm{O} \mathrm{cm}$. Mean final filling pressure was $68 \mathrm{H} 2 \mathrm{O} \mathrm{cm}$ before treatment and $34 \mathrm{H} 2 \mathrm{O} \mathrm{cm}$ after. Mean reflex volume was $717.33 \mathrm{ml}$ before and $310.8 \mathrm{ml}$ after. Finally, mean post void residual before BTX-A was 129.6 $\mathrm{ml}$ whereas post-BTX-A was $345.9 \mathrm{ml}$.

CONCLUSIONS: The injection of $300 \mathrm{U}$ of type $A$ botulin toxin in the detrusor muscle has demonstrated to be effective and safe for the treatment of refractory neurogenic hyperactive detrusor. There were statisticaIly significant differences in the urodynamic parameters before and after the injection. There were no significant adverse events.

Keywords: Botulin toxin. Neurogenic bladder. Neurogenic hyperactive detrusor. Refractory neurogenic hyperactive detrusor. Detrusor injection.

\section{INTRODUCCIÓN}

El correcto tratamiento de la vejiga hiperactiva neurogénica secundaria a lesiones medulares suprasacrales constituye uno de los principales pilares en el manejo de los pacientes lesionados medulares. De su correcto tratamiento dependen aspectos funda- mentales para la vida como la suficiencia renal pero también la calidad de vida podrá verse sensiblemente mejorada, con el impacto psicosocial que ella conlleva. En su tratamiento son utilizados habitualmente agentes anticolinérgicos con excelentes resultados, aunque no son infrecuentes las fallas de tratamiento ya sea por falta de eficacia o por intolerables efectos adversos como la sequedad de boca, constipación, visión borrosa o trastornos cognitivos. En aquellos pacientes refractarios a la terapia anticolinérgica investigamos la eficacia de la toxina botulínica.

La intoxicación por toxina botulínica fue descripta por primera vez a finales de 1700 en Alemania. Pero recién en 1897 van Ermengem aisló la bacteria anaerobia obligada formadora de esporas y la llamó Clostridium botulinum. En 1923 Dickson y Shevky demostraron que la toxina botulínica (BTX) dañaba la transmisión nerviosa en la terminal muscular (1). Y en 1949 Burgen demostró que la parálisis era causada por la inhibición de la liberación de acetilcolina (2). En la década de los 80 se introduce la Toxina botulínica tipo A para el tratamiento del estrabismo. Desde entonces su uso se ha ampliado desde el tratamiento de las distonías focales y segmentarias, blefaroespasmo, distonía cervical, distonía oromandibular, distonía laringea, espasmo hemifacial, hiperhidrosis, temblores, tics, acalasia, espasmo anal, vaginismo, dolores miofasciales, y sin dejar de lado su amplio uso en cirugía estética (3-5).

Durante los años 90 se introduce la aplicación en espasticidad por parálisis cerebral, traumatismo craneoencefálico, accidentes cerebrovasculares, esclerosis múltiple y lesión medular $(6,7)$. Y a partir de finales de la década del 90 su uso se extiende al campo de la urología. Las primeras publicaciones con aplicaciones experimentales en urología fueron publicadas en 1988 y 1990 por Dykstra $(8,9)$, pero sus trabajos fueron realizados en pacientes que padecían lesiones medulares con disinergia detrusor-esfínter externo, y aplicando un preparado de toxina experimental solamente al esfínter. Recién en 1996 Schurch (10) y en 1998 Petit (11) repiten las experiencias de Dykstra en el mismo tipo de pacientes pero con preparados comerciales en venta en el mercado. En el año 2000 Schurch (12) publica sus investigaciones aplicando Botox $\AA$ tanto en esfínter externo como en vejiga, y de ese modo se aumenta el espectro de patologías urológicas a tratar. Nosotros realizamos las primeras aplicaciones de toxina botulínica tipo $A$ en preparado comercial Botox $\circledast$ aplicándolas tanto en vejiga como en esfínter externo a partir de 2001 .

El objetivo propuesto es la evaluación de la eficacia de la inyección intradetrusorial de toxina bo- 
tulínica tipo $A$ en pacientes con detrusor hiperactivo neurogénico refractario, en la capacidad cistomanométrica máxima, en el volumen de reflejo asociado a disrreflexia autonómica, en la acomodación del detrusor $y$ en el residuo postmiccional.

\section{MATERIAL Y MÉTODOS}

Se realizó un estudio prospectivo en 16 pacientes con detrusor hiperactivo neurogénico refractario a la terapia anticolinérgica. Se estableció como criterio de inclusión a pacientes con diagnóstico de detrusor hiperactivo secundario a lesión neurológica diagnosticado mediante estudio urodinámico (cistometría y estudio de presión/flujo) con medición de la actividad bioeléctrica del piso perineal medida con electrodos de contacto y refractarios a la terapia anticolinérgica. Se definió como refractarios a aquellos pacientes que no observaron mejoría suficiente en los episodios de incontinencia de orina, crisis de disrreflexia autonómica o número de cateterismos intermitentes luego de la administración de dosis máxima de oxibutinina, tolterodina y trospium. Los criterios de exclusión fueron miastenia gravis, embarazo, lactancia y cualquier enfermedad concomitante seria que pudiere afectar la vida de los pacientes.

Todos los pacientes completaron evaluación urológica consistente en anamnesis, examen físico, diario miccional por $48 \mathrm{hs}$, orina completa, urocultivo, función renal, ecografía renal y vesical, y estudio de Urodinamia con medición de actividad bioeléctrica de piso perineal. La Urodinamia fue realizada según las "Buenas Prácticas Urodinámicas" (13) recomendadas por la Sociedad Internacional de la Continencia con equipo de Urodinamia ECUD® de Medware Argentina. Los parámetros urodinámicos registrados incluyeron la capacidad cistomanométrica máxima, la acomodación vesical, el volumen de reflejo, la presión máxima del detrusor y el residuo postmiccional. Todos los métodos, definiciones y unidades utilizadas son conformes a las estandarizaciones recomendadas por la Sociedad Internacional de la Continencia (14). También se realizó medición de tensión arterial y pulso durante el estudio urodinámico previo al llenado, durante el mismo y una vez vaciada la vejiga como registro de crisis de disrreflexia autonómica. La medicación anticolinérgica se interrumpió por lo menos 2 semanas antes de la evaluación urodinámica previa.

Se realizaron estudios urodinámicos 30 a 60 días previos al procedimiento. Bajo cobertura antibiótica según antibiograma de cada paciente se realizó la inyección de $300 \mathrm{Um}$ de toxina botulínica tipo A Botox® según la técnica propuesta por Schurch y cols. (12). El procedimiento de inyección se llevó a cabo en posición de litotomía, bajo neuroleptoanalgesia y mediante la utilización de aguja Prostaject $\circledast$ de alcoholización prostática de $\mathrm{AMS} \circledast$ bajo visión directa con cistoscopio Storz con camisa $20 \mathrm{Fr}$. Se inyectaron $300 \mathrm{Um}$ diluidos en $30 \mathrm{ml}$ de solución fisiológica divididos en 30 sitios de punción de $1 \mathrm{ml}$ cada una repartidas en todas las caras de la vejiga pero respetando el trígono con el fin de evitar la posibilidad de reflujo vesicoureteral. Monitoreo cardiovascular fue realizado durante el procedimiento. Se realizó una urodinamia de control 30 a 45 días posteriores a la inyección de toxina botulínica.

\section{RESULTADOS}

Entre noviembre de 2002 y junio de 2005 se inyectaron 16 pacientes (5 mujeres y 11 hombres). La edad media de los pacientes fue de 39,5 años (22 a 63 años). Todos los pacientes presentaban diagnóstico urodinámico de detrusor hiperactivo neurogénico. Nueve de ellos con disinergia detrusor esfínter externo. Diez de ellos se les realizaba vaciado vesical con cateterismo asistido y 1 de ellos realizaba vaciado vesical con maniobras de provocación. 5 pacientes presentaban micción refleja.

Todos los pacientes presentaban diagnóstico neurológico de lesión medular suprasacral (Tabla I). Según la clasificación de la American Spinal Injury Association 13 pacientes presentaban lesión completa $\circ$ ASIA A por trauma raquimedular (TRM) y 2 presentaban lesión incompleta ASIA D uno por TRM y otro por mielitis transversal (MT) y un paciente ASIA E. 7 pacientes presentaban crisis de disrreflexia autonómica como respuesta a la vejiga llena definida como aumento de la tensión arterial y/o presencia de taquicardia, sudoración, rubor, piloerección o cefaleas, observadas durante la evaluación urodinámica inicial.

El tiempo de inyección de la toxina botulínica por vía endoscópica no superó los 30 minutos en ninguno de los casos. Como complicación se observó hematuria macroscópica en 3 casos los cuales cedieron espontáneamente. $Y$ ningún episodio de hematuria se prolongó por más de 24 hs. Ninguna de ellas requirió algún procedimiento adicional. Tampoco se observaron otras complicaciones.

El análisis estadístico se realizó con el programa STATISTIX 7.0 (Analytical software USA.) Se tomaron en cuenta cinco parámetros:

Capacidad cistométrica máxima (CCMX); presión máxima del detrusor (PMXDET); presión de 
fin de llenado (PFLL); volumen reflejo (VOL) y residuo postmiccional (RPM) (Tablas II y III).

Se analizó la normalidad de las variables en estudio; luego se seleccionaron las pruebas estadísticas según fuera el caso para análisis comparativo. Se utilizó:

- Para las variables con distribución normal: T TEST DE STUDENT PARA MUESTRAS APAREADAS.

- Para las variables sin distribución normal: TEST DE LA MEDIANA.

Para CCMX, PFLL y VOL se utilizó el TTEST DE STUDENT PARA MUESTRAS APAREADAS, mostrando diferencias estadísticamente significativas entre valores pre y post-Botox con valores de $\mathrm{P}$ de 0.0000 , 0.001 y 0.0000 respectivamente.

Para PMXDET y RPM se utilizó TEST DE LA MEDIANA, mostrando también diferencias estadística- mente significativas entre valores pre y post-Botox con valores de $\mathrm{P}$ de 0.0000 y 0.001 respectivamente.

Todos los pacientes que presentaban crisis de disrreflexia autonómica previas manifestaron disminución de dichos eventos, los cuales influyeron fuertemente en la mejoría de la calidad de vida.

No se observaron efectos adversos esperables relacionados con la toxina como disfagia, diplopía, paresias, astenia o adinamia.

\section{DISCUSIÓN}

La vejiga hiperactiva actualmente es tratada con agentes antimuscarínicos con buenos resultados desde los años setenta (15). Si bien agentes como la hiosciamina y la propantelina han sido abandonados, drogas como la oxibutinina, tolterodina, darifenacina, trospium y solifenacina están actualmente en uso ya que presentan mayor tolerabilidad ya sea

TABLA I.

\begin{tabular}{|c|c|c|c|c|c|c|}
\hline $\mathbf{N}^{\circ}$ & Nombre & Edad & Sexo & Lesión & Nivel & ASIA \\
\hline 1 & CM & 26 & $f$ & TRM & C4-C5 & A \\
\hline 2 & RP & 22 & $f$ & TRM & C6 & A \\
\hline 3 & RA & 56 & $\mathrm{~m}$ & TRM & C5-C6 & A \\
\hline 4 & MC & 37 & $\mathrm{~m}$ & TRM & D9 & D \\
\hline 5 & DM & 53 & $\mathrm{~m}$ & TRM & C4 & A \\
\hline 6 & MW & 24 & $\mathrm{~m}$ & TRM & C4 & A \\
\hline 7 & AV & 51 & $\mathrm{f}$ & TRM & D9 & A \\
\hline 8 & RG & 63 & $\mathrm{~m}$ & TRM & D9 & A \\
\hline 9 & SI & 40 & $\mathrm{~m}$ & TRM & C4 & A \\
\hline 10 & DL & 20 & $\mathrm{f}$ & TRM & C4-C5 & A \\
\hline 11 & EA & 38 & $\mathrm{f}$ & QT & L4-L5 & $\mathrm{E}$ \\
\hline 12 & CL & 57 & $\mathrm{~m}$ & TRM & D6-D8 & A \\
\hline 13 & SM & 39 & $\mathrm{~m}$ & TRM & L3 & A \\
\hline 14 & JB & 25 & $\mathrm{~m}$ & TRM & C6-C7 & A \\
\hline 15 & JF & 37 & $\mathrm{~m}$ & MT & L4-L5 & D \\
\hline 16 & EP & 44 & $\mathrm{~m}$ & TRM & D8 & A \\
\hline
\end{tabular}


TABLA II. VALORES PRE-BOTOX.

\begin{tabular}{|l|c|c|c|c|}
\hline & Media & IC < 95\% & IC > 95\% & DS \\
\hline CCMX (ml.) & 204.73 & 150 & 259.4 & 98.8 \\
\hline PMXDET (cm. de agua) & 91.4 & 66.4 & 116.4 & 45 \\
\hline PFLL (cm de agua) & 68 & 48.9 & 87 & 34.4 \\
\hline VOL (ml.) & 111.33 & 82.9 & 139.6 & 51.2 \\
\hline RPM (ml.) & 129.6 & 66.5 & 192.6 & 113.9 \\
\hline
\end{tabular}

por su mecanismo de acción o por su formulación (16-19). Sin embargo algunos pacientes son refractarios a estos fármacos. Terapias conductuales, combinación de drogas, kinesioterapia de piso pelviano deben ser incorporadas a ésta línea de tratamiento, pero no siempre ofrecen los resultados deseados. Es por ello que la vejiga hiperactiva refractaria constituye un cuadro de difícil abordaje. Además los efectos adversos que los agentes antimuscarínicos provocan son una de las causas más frecuentes de abandono de tratamiento. La sequedad de boca, constipación y visión borrosa son los efectos adversos más frecuentes. Pero los efectos sobre el sistema nervioso central como los trastornos cognitivos, y sobre el corazón como la arritmia constituyen 2 efectos de no menor importancia (20). Además son contraindicadas en glaucoma de ángulo estrecho, retención gástrica y deben ser utilizadas con precaución en pacientes añosos, en reflujo gastroesofágico, constipación, trastornos cognitivos y demencia.

Los agentes endovesicales como la capsaicina y la resinferotoxina han demostrado en diversos ensayos clínicos, ser efectivas en el tratamiento de la vejiga hiperactiva, pero los trastornos sensitivos de dolor, ardor, sofocos de calor, disrreflexia autonómica y hematuria han sido el principal obstáculo para la aceptación de ésta terapia (21).

Mientras tanto terapias más invasivas como la neuroestimulación sacra con rizotomías sacras, miomectomía vesical, cirugías de denervación vesical y cistoplastía de ampliación se mantienen como tratamientos de la vejiga hiperactiva refractaria. Pero dado el carácter de irreversibilidad de éstos procedimientos hace que los pacientes sean más reacios a aceptar éstas indicaciones.

En éste contexto la inyección de BTX-A ofrece una terapia eficaz, de baja morbilidad y reversible.

Las primeras aplicaciones en vejiga fueron realizadas por Schurch y cols. en el año 2000. En sus investigaciones demostró el aumento de la media de la capacidad cistomanométrica máxima (capacidad vesical) de 296 a $480 \mathrm{ml}$ y una disminución en la media de la presión de detrusor máxima miccional de 65 a $35 \mathrm{~cm}$ de agua en 21 pacientes inyectados

TABLA III. VALORES POST-BOTOX.

\begin{tabular}{|l|c|c|c|c|}
\hline & Media & IC <95\% & IC > 95\% & DS \\
\hline CCMX (ml.) & 381.87 & 320 & 443.7 & 111.6 \\
\hline PMXDET (cm. de agua) & 41.2 & 30 & 52.3 & 20.1 \\
\hline PFLL (cm de agua) & 68 & 48.9 & 87 & 34.4 \\
\hline VOL (ml.) & 310.8 & 262.6 & 358.9 & 86.8 \\
\hline RPM (ml.) & 345.9 & 274.8 & 417 & 128.3 \\
\hline
\end{tabular}


con 200 y $300 \mathrm{U}$ de BTX-A (22). De 19 pacientes, 17 se mantuvieron continentes a las 6 semanas de seguimiento. Los 2 pacientes incontinentes resultaron por utilizar 200 en lugar de $300 \mathrm{U}$ de BTX-A. Y en 11 pacientes se mantuvieron los parámetros urodinámicos y de continencia durante 36 semanas. Desde entonces numerosos estudios han confirmado éstas experiencias iniciales en series de hasta 200 pacientes con seguimientos de 3 y 6 meses (23). Y en nuestra serie personal con 16 pacientes adultos observamos un aumento de la capacidad cistomanométrica media de $205 \mathrm{ml}$ a $382 \mathrm{ml}$, la presión máxima del detrusor media disminuyó de 91 a $41 \mathrm{~cm}$ de agua, el volumen de reflejo (el volumen al cual se observa la primer contracción no inhibida del detrusor) aumentó de $111 \mathrm{ml} \mathrm{a} 310 \mathrm{ml}$. Mientras que el residuo postmiccional aumentó de $130 \mathrm{ml}$ a $346 \mathrm{ml}$. De éste modo se logró una significativa mejoría en la calidad de vida de los pacientes ya que se logró disminuir el número de pañales y los episodios de incontinencia. Además debido a que el volumen de reflejo (primera contracción no inhibida del detrusor) se correlaciona con las crisis de disrreflexia autonómica, al aumentarse dicho volumen, aquellos pacientes que padecían las crisis lograron que éstas ocurran más tardíamente y con menor intensidad, y éste se constituyó en otro elemento fundamental a la hora de evaluar la calidad de vida. Y como aspecto primordial al disminuir la presión máxima del detrusor se logra disminuir ostensiblemente el riesgo de reflujo vesicoureteral y concomitante el riesgo potencial de insuficiencia renal.

El uso de la toxina también se ha extendido a los trastornos no neurogénicos del almacenamiento como la vejiga hiperactiva idiopática y el síndrome de urgencia y frecuencia miccional. Radziszewski reportó en el 2001 una disminución de la frecuencia y urgencia miccional en 4 de 7 pacientes (24). Desde entonces numerosas publicaciones avalan éste uso. Recientemente Rajkumar y cols. evaluaron en forma prospectiva a 15 mujeres con detrusor hiperactivo idiopático encontrando mejoría sintomática en 14 de ellas hasta 24 semanas luego de la inyección (25).

Con respecto a la técnica se recomienda el uso de 200 a $300 \mathrm{U}$ de toxina inyectada endoscópicamente en 30 o 40 sitios de punción mediante una dilución de $1 \mathrm{ml}$ equivalente a $10 \mathrm{U}$. distribuidos uniformemente. Algunos autores recomiendan evitar el trígono con el fin de evitar el reflujo vesicoureteral, pero ésta complicación no ha sido probada.

Los efectos adversos reportados fueron escasos. Del Poppolo reportó hipostenia en 5 de 61 pacientes, los cuales desaparecieron entre las 2 a 4 semanas posteriores (26). Wyndaele y Van Dromme lo reportaron en 2 pacientes con lesión medular (27).

\section{CONCLUSIÓN}

La inyección de $300 \mathrm{U}$ de toxina botulínica tipo $A$ en el detrusor ha demostrado ser eficaz y segura para el tratamiento del detrusor hiperactivo neurogénico refractario. Los parámetros urodinámicos analizados: Capacidad cistométrica máxima (CCMX); presión máxima del detrusor (PMXDET); presión de fin de llenado (PFLL); volumen reflejo (VOL) y Residuo postmiccional (RPM), presentaron diferencias estadísticamente significativas antes y después de la inyección. No se presentaron efectos adversos significativos. Si bien deben analizarse los resultados con precaución podemos decir que nos encontramos frente a una droga que será de gran utilidad para tratar estas afecciones de difícil tratamiento.

\section{NOTA}

Parte de este trabajo fue realizado con fondos provenientes de la beca de investigación otorgada por la Confederación Americana de Urología (CAU).

\section{BIBLIOGRAFÍA y LECTURAS RECOMENDADAS (*lectura de interés $y^{* *}$ lectura fundamental)}

1. DICKSON, E.C.; SHEVKY, R.: "Botulism: Studies on the manner in which the toxin of Clostridium botulinum acts upon the body. II. The effect on the voluntary nervous system". J. Exp. Med., 38: 327, 1923.

2. BURGEN, A.S.V.; DICKENS, F.; ZATMAN, L.J.: "The action of botulinum toxin on the neuromuscular junction". J. Physiol. (Lond)., 109: 10, 1949.

3. GRANDAS, F.: "Aplicaciones clínicas de la toxina botulínica”. Neurología, 10: 224, 1995.

4. KESSLER, K.R.; SKUTTA, M.: "Long-term treatment of cervical dystonia with botulinum toxin A: Efficacy, safety, and antybody frequency. German Dystonia Study Group”. J. Neurol., 246: 265, 1999.

5. SALOMON, B.A.; HAYMAN, R.: "Botulinum toxin type A for palmar and digital hyperhidrosis". J. Am. Acad. Dermatol., 42: 1026, 2000.

6. BRIN, F.: "Dosing, administration and treatment algorythm for use of botulinum toxin A for adultonset spasticity". Muscle Nerve, 6: 208, 1997.

7. EDGAR, T.S.: "Clinical utility of botulinum toxin in the treatment of cerebral palsy: Comprehensive review". J. Child. Neurol., 16: 37, 2001.

8. DYKSTRA, D.D. y cols.: "Effects of botulinum A toxin on detrusor-sphincter dyssynergia in spinal cord injury patients". J. Urol., 139: 919, 1988. 
9. DYKSTRA, D.D.; SIDI, A.: “Treatment of detrusor-sphincter dyssynergia with botulinum A toxin: A double blind study". Arch. Phys. Med. Rehabil., 71: 24, 1990.

*10. SCHURCH, B. y cols.: "Botulinum A toxin as a treatment of detrusor-sphincter dyssynergia: A prospective study in 24 spinal cord injury patients". J. Urol., 155: 1023, 1996.

11. PETIT, H. y cols.: "Botulinum A toxin treatment for detrusor-sphincter dyssynergia in spinal cord disease". Spinal Cord., 36: 91, 1998.

**12. SCHURCH, B. y cols.: "Botulinum-A toxin for treating detrusor hyperreflexia in spinal cord injured patients: A new alternative to anticholinergic drugs? Preliminary results". J. Urol., 164: 692, 2000.

13. SCHÄFER, W.; ABRAMS, P.; LIAO, L. y cols.: "Good Urodynamic Practices: Uroflowmetry, Filling Cystometry, and Pressure-Flow studies. Neurourology and Urodynamics, 21: 261, 2002.

14. ABRAMS, P.; CARDOZO, L.; FALL, M. y cols.: "The Standardisation of terminology of Lower Urinary Tract Function: Report from the Standarization Sub-committee of the International Continence Society". Neurourol. Urodyn. 21: 167, 2002.

15. DIOKNO, A.; LAPIDES, J.: “Oxybutynin: A new drug with analgesic and anticholinergic properties". J. Urol., 108: 307, 1972.

16. LARSSON, G.; HALLEN, B.; NILVER-

16. BRANT, L.: "Tolterodine in the treatment of overactive bladder: Analysis of the pooled phase II efficacy and safety data”. Urology, 53: 990, 1999.

17. ROSARIO, D.; CUTINHA, P.; CHAPPLE, C. y cols.: "The effects of single dose darfenacin on cystometric parameters and salivary flow in patients with urge incontinence secondary to detrusor instability". Eur. Urol., 30: 240, 1996.

18. CHAPPLE, C.R. y cols.: "Randomised, doubleblind placebo and tolterodine controlled trial of the once-daily antimuscarinic agent soifenacin in patients with symptomatic overactive bladder". BJU International, 93: 303, 2004.

19. ZINNER, N. y cols.: "Trospium chloride improves overactive bladder symptoms: A multicenter phase III trial". J. Urol., 71: 311, 2004.

20. KATZ, I.R.; SANDS, L.P.; BILKER, W. y cols.: "Identification of medications that cause cognitive impairment in older people: The case of oxybutynin chloride". J. Am. Geriatr. Soc., 46: 8, 1998.

21. CHANCELLOR, M.B.; DE GROAT, W.C.: "Intravesical capsaicin and resiniferatoxin therapy: Spicing up the ways to treat the overactive bladder". J. Urol., 162: 3, 1999.

**22. SCHURCH, B. y cols.: "Botulinum-A toxin for treating detrusor hyperreflexia in spinal cord injured patients: A new alternative to anticholinergic drugs? Preliminary results". J. Urol., 164: 692, 2000.

*23. REITZ, A.; STOHRER, M.; KRAMER, G. y cols.: "European experience of 200 cases treated with botulinum-A toxin injections into the detrusor muscle for urinary incontinence due to neurogenic detrusor overactivity". Eur. Urol., 45: 510, 2004.

*24. RADZISZEWSKI, P.; DOBRONSKI, P.; BORKOWSKI, A.: "Treatment of the non-neurogenic storage and voiding disorders with the chemical denervation caused by botulinum". Neurourol. Urodyn., 20: 410, 2001.

25. RAJKUMAR, G.N. y cols.: "A prospective study to evaluate the safety, tolerability, efficacy and durability of response of intravesical injection of botulinum toxin type A into detrusor muscle in patients with refractory idiopathic detrusor overactivity”. BJU Int., 97: 413, 2006.

26. DEL POPPOLO, G.: "Botulinum A toxin in the treatment of detrusor hyperreflexia”. Neurourol. Urodyn., 20: 522, 2001.

27. WYNDAELE, J.J.; VAN DROMME, S.A.: "Muscular weakness as side effect of botulinum toxin injection for neurogenic detrusor overactivity". Spinal Cord., 40: 599, 2002. 